Bull. Korean Math. Soc. 49 (2012), No. 6, pp. 1199-1211

http://dx.doi.org/10.4134/BKMS.2012.49.6.1199

\title{
ON UNIVERSAL COVERINGS OF LIE TORI
}

\author{
VALiolLah KhaLiLI
}

\begin{abstract}
In this paper we give an introduction to the theory of universal central extensions of perfect Lie algebras. In particular, we will provide a model for the universal coverings of Lie tori and we show that automorphisms and derivations lift to the universal coverings. We also prove that the universal covering of a Lie $\Lambda$-torus of type $\Delta$ is again a Lie $\Lambda$-torus of type $\Delta$.
\end{abstract}

\section{Introduction}

Central extensions play an important role in the theory of Lie algebras. Universal central extension of Lie algebras over rings were described in [23, §1], later references on universal central extensions are [12, §1], [15, 1.9], [24, 7.9] or [21]. Central extensions in the category of certain topological Lie algebras are studied in [17]. Garland studies universal central extensions of Lie algebras over fields $[12, \S 1]$. In particular, he constructs a model of universal central extension of a perfect Lie algebra, using the universal 2-cocycle, which is different from the Van der Kallen's model (see $[23, \S 1]$ ). Our construction for universal central extensions of Lie tori is essentially the Van der Kallen's model.

Lie tori play a critical role in the theory of extended affine Lie algebras which are natural generalization of finite dimensional simple Lie algebras and affine Lie algebras. Yoshii and Neher were interested in Lie tori primarily because of the connection between Lie tori and extended affine Lie algebras ([26] and [19]). The centerless core of an extended affine Lie algebra is a centerless of Lie torus and conversely any centerless Lie torus is the centerless core of an extended affine Lie algebra [26, Theorem 7.3]. Extended affine Lie algebras are defined axiomatically by Alison, Azam, Berman, Gao and Pianzola in [1]. The various classes of these Lie algebras have been investigated in many papers (see $[4,6,13,14,20,25,27]$ and [16]). Lie tori as well as extended affine Lie

Received June 28, 2010

2010 Mathematics Subject Classification. 17B67.

Key words and phrases. root graded Lie algebras, extended affine Lie algebras and Lie tori.

(C)2012 The Korean Mathematical Society 
algebras are also defined axiomatically, which are the certain root graded Lie algebras.

The universal central extensions of root-graded Lie algebras are determined in [2], [3] and [8]. Recently, in [28] and [7] the authors have given a finite presentation for the universal covering of the centerless core of a Lie torus of type $X, X \neq A_{l}, C_{l}, B C_{l}$. Namely, by using the recognition and structural theorem(s) for root graded Lie algebras and their universal coverings, they were able to select a finite set of generators and deduce a certain set of relations among them for presentation the universal covering of a Lie torus of type $X$, $X \neq A_{l}, C_{l}, B C_{l}$.

One of our aims in this note is to give natural construction for universal covering of Lie tori, with emphasis on the general part of the theory, rather than on special type of Lie tori. More precisely, our construction uses the model of the universal central extension of perfect Lie algebras by Van der Kallen's method $[23, \S 1]$. The purpose of this note is to give an information about the structure for the universal covering of a Lie $\Lambda$-torus of type $\Delta$, where $\Lambda$ is an abelian group and $\Delta$ is an irreducible finite root system.

To close this introduction, we briefly outline the contents of the paper. In Section 1 , we recall the definition of Lie $\Lambda$-torus of type $\Delta$ and some facts that we will need in the sequel. We also give an introduction to the theory of universal central extension for perfect Lie algebras and record some properties of it. In Section 2, we provide a construction for universal covering of Lie tori, by using Van der Kallen's model. We finish this section by proving that automorphisms and derivations of Lie tori lift to the universal central extension. Section 3 contains the structure of the universal covering of a Lie $\Lambda$-torus of type $\Delta$. More precisely we show that the universal covering of a Lie $\Lambda$-torus of type $\Delta$ satisfies in all axioms of Lie tori and is again a Lie $\Lambda$-torus of type $\Delta$.

\section{Basic definitions and terminology}

Throughout this paper we will assume that $\Lambda$ is an abelian additive group and $\mathbb{F}$ is a field of characteristic zero, unless otherwise mentioned, all vector spaces and all algebras are considered over $\mathbb{F}$.

\subsection{Lie tori}

First we introduce a notion of root graded Lie algebra. Specially, we consider certain root graded Lie algebra called Lie torus, which was first introduced in [26] and [27].

Let $\Delta$ be a finite irreducible root system (which is contains, zero and not necessarily reduced) as in [10, Chap VI]. For each root $\alpha \in \Delta^{\times}:=\Delta \backslash\{0\}$, let $\check{\alpha}$ be the corresponding coroot such that $\langle\beta, \check{\alpha}\rangle$ be the Cartan integer for all $\beta \in \Delta$. Set $\Delta_{\text {ind }}:=\{0\} \cup\{\alpha \in \Delta \mid \alpha / 2 \notin \Delta\}$. We denote the root lattice of $\Delta$ by $Q(\Delta)$. 
Let $\mathcal{G}$ be a finite-dimensional split simple Lie algebra over $\mathbb{F}$ with root space decomposition $\mathcal{G}=\mathcal{H} \oplus\left(\bigoplus_{\alpha \in \Delta_{i n d}} \mathcal{G}_{\alpha}\right)$ relative to a split Cartan subalgebra $\mathcal{H}$. Every finite root system $\Delta$ is one of the reduced root system $\Delta_{i n d}$ or is a non reduced root system of type $B C_{l}$.

Definition 1.1. Let $\mathcal{L}$ be a Lie algebra over a field $\mathbb{F}$, and let $\Delta$ be a finite irreducible root system. Then $\mathcal{L}$ is said to be graded by the root system $\Delta$ or to be $\Delta$-graded if

(i) $\mathcal{L}$ contains as a subalgebra, a finite dimensional split simple Lie algebra $\mathcal{G}$, called the grading subalgebra with root system $\Delta_{\text {ind }}$ relative to a split Cartan subalgebra $\mathcal{H}$.

(ii) $\mathcal{L}$ has a decomposition $\mathcal{L}=\bigoplus_{\alpha \in \Delta} \mathcal{L}_{\alpha}$, where $\mathcal{L}_{\alpha}=\{x \in \mathcal{L}:[h, x]=$ $\alpha(h) x$ for all $h \in \mathcal{H}\}$.

(iii) $\mathcal{L}_{0}=\sum_{\alpha \in \Delta \times}\left[\mathcal{L}_{\alpha}, \mathcal{L}_{-\alpha}\right]$.

(iv) Either $\Delta$ is reduced and equals the root system $\Delta_{\text {ind }}$ of grading pair $(\mathcal{G}, \mathcal{H})$ or $\Delta$ is of type $B C_{l}$ and $\Delta_{i n d}$ is of type $B_{l}, C_{l}$, or $D_{l}$.

The above definition is due to Berman and Moody [9] for the case $\Delta=\Delta_{\text {ind }}$. The extension to the nonreduced root systems $B C_{l}$ was developed by Alison, Benkart and Gao in [3] for $l \geq 2$ and by Benkart and Smirnov in [8] for $l=1$.

Definition 1.2. A Lie algebra $\mathcal{L}$ is a Lie $\Lambda$-torus of type $\Delta$ if the following axioms hold:

(LT1) $\mathcal{L}$ has a grading by $(Q(\Delta) \oplus \Lambda)$, of the form

$$
\mathcal{L}=\bigoplus_{\alpha \in \Delta} \bigoplus_{\lambda \in \Lambda} \mathcal{L}_{\alpha}^{\lambda}, \quad\left[\mathcal{L}_{\alpha}^{\lambda}, \mathcal{L}_{\beta}^{\mu}\right] \subset \mathcal{L}_{\alpha+\beta}^{\lambda+\mu}, \text { satisfying } \mathcal{L}_{\alpha}^{\lambda}=0 \text { if } \alpha \notin \Delta .
$$

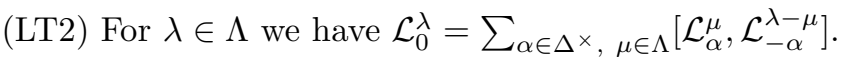

(LT3) For $\alpha \in \Delta^{\times}$and $\lambda \in \Lambda$ we have:

(i) $\operatorname{dim} \mathcal{L}_{\alpha}^{\lambda} \leq 1$, with $\operatorname{dim} \mathcal{L}_{\alpha}^{0}=1$ if $\alpha \in \Delta_{\text {ind }}$,

(ii) if $\operatorname{dim} \mathcal{L}_{\alpha}^{\bar{\lambda}}=1$, then there exists $\left(e_{\alpha}^{\lambda}, f_{\alpha}^{\lambda}\right) \in \mathcal{L}_{\alpha}^{\lambda} \times \mathcal{L}_{-\alpha}^{-\lambda}$ such that $h_{\alpha}^{\lambda}:=$ $\left[e_{\alpha}^{\lambda}, f_{\alpha}^{\lambda}\right] \in \mathcal{L}_{0}^{0}$ acts on $x_{\beta}^{\mu} \in \mathcal{L}_{\beta}^{\mu}(\beta \in \Delta, \mu \in \Lambda)$ by

$$
\left[h_{\alpha}^{\lambda}, x_{\beta}^{\mu}\right]=\langle\beta, \check{\alpha}\rangle x_{\beta}^{\mu} .
$$

(LT4) $\Lambda=\left\langle\left\{\lambda \in \Lambda \mid \mathcal{L}_{\alpha}^{\lambda} \neq 0\right.\right.$ for some $\left.\left.\alpha \in \Delta\right\}\right\rangle$.

The type of $\mathcal{L}$ is by definition the type of the finite root system $\Delta$, and the rank of $\Lambda$ is called the nullity of $\mathcal{L}$. Finally, $\mathcal{L}$ is called centerless if the center $Z(\mathcal{L})$ of $\mathcal{L}$ is zero.

Let $\mathcal{L}=\bigoplus_{\alpha \in \Delta} \bigoplus_{\lambda \in \Lambda} \mathcal{L}_{\alpha}^{\lambda}$ be a Lie $\Lambda$-torus of type $\Delta$. It follows from (LT1) that $\mathcal{L}$ has a grading by $Q(\Delta)$ as

$$
\mathcal{L}=\bigoplus_{Q(\Delta)} \mathcal{L}_{\alpha}, \quad \text { where } \quad \mathcal{L}_{\alpha}=\bigoplus_{\lambda \in \Lambda} \mathcal{L}_{\alpha}^{\lambda},
$$


and also $\mathcal{L}$ is $\Lambda$-graded by

$$
\mathcal{L}=\bigoplus_{\lambda \in \Lambda} \mathcal{L}^{\lambda}, \quad \text { where } \quad \mathcal{L}^{\lambda}=\bigoplus_{\alpha \in \Delta} \mathcal{L}_{\alpha}^{\lambda}
$$

The two gradings are compatible by $\mathcal{L}_{\alpha}^{\lambda}:=\mathcal{L}^{\lambda} \cap \mathcal{L}_{\alpha}$ for all $\alpha \in \Delta$ and $\lambda \in \Lambda$.

If we fix an ordered basis $\left\{\alpha_{i} \mid 1 \leq i \leq \ell\right\}$ of $\Delta$. By (LT3)(ii), for each $\alpha \in \Delta_{\text {ind }}$ there exists $\left(e_{\alpha}, f_{\alpha}\right) \in \mathcal{L}_{\alpha}^{0} \times \overline{\mathcal{L}}_{-\alpha}^{0}$, such that $\left(e_{\alpha}, h_{\alpha}:=\left[e_{\alpha}, f_{\alpha}\right], f_{\alpha}\right)$ is an $\mathfrak{s l}_{2}^{(\alpha)}$-triple. The Lie subalgebra of $\mathcal{L}^{0}$ generated by $e_{\alpha}, h_{\alpha}, f_{\alpha}$ is isomorphic to $\mathfrak{s l}_{2}(\mathbb{F})$. Let $\mathcal{G}$ be the subalgebra of $\mathcal{L}^{0}$ generated by $\left\{e_{\alpha_{i}}, h_{\alpha_{i}}, f_{\alpha_{i}} \mid 1 \leq i \leq \ell\right\}$ and $\mathcal{H}=\sum_{i=1}^{\ell} \mathbb{F} h_{\alpha_{i}}$. It is showed in [5] that $\mathcal{G}$ is a finite dimensional split simple Lie subalgebra of the Lie torus $\mathcal{L}$ with splitting Cartan subalgebra $\mathcal{H}$ and root system $\Delta_{\text {ind }}$, relative to $\mathcal{H}$. Then $\mathcal{L}$ is a $(\Delta, \Lambda)$-graded Lie algebra with grading pair $(\mathcal{G}, \mathcal{H})$, in the sense of $[26]$.

Definition 1.5. Let $\mathcal{L}$ be a Lie algebra. A central extension of $\mathcal{L}$ is a pair $(\tilde{\mathcal{L}}, \pi)$ consisting of a Lie algebra $\tilde{\mathcal{L}}$ and a surjective Lie algebra homomorphism $\pi: \tilde{\mathcal{L}} \longrightarrow \mathcal{L}$ whose kernel lies in the center $Z(\tilde{\mathcal{L}})$ of $\tilde{\mathcal{L}}$. A central extension $(\tilde{\mathcal{L}}, \pi)$ of $\mathcal{L}$ is called a covering (or a cover) if $\tilde{\mathcal{L}}$ is perfect $([\tilde{\mathcal{L}}, \tilde{\mathcal{L}}]=\tilde{\mathcal{L}})$. A homomorphism from a central extension $(\tilde{\mathcal{L}}, \pi)$ of $\mathcal{L}$ to another central extension $(\check{\mathcal{L}}, \check{\pi})$ of $\mathcal{L}$ is a Lie algebra homomorphism $\varphi: \tilde{\mathcal{L}} \longrightarrow \check{\mathcal{L}}$ which is satisfies $\check{\pi} \circ \varphi=\pi$. A central extension $(\tilde{\mathcal{L}}, \pi)$ of $\mathcal{L}$ is called a universal central extension if there exists a unique homomorphism from $(\tilde{\mathcal{L}}, \pi)$ to any other central extension of $\mathcal{L}$.

Remark 1.6. (a) By a central extension $(\tilde{\mathcal{L}}, \pi)$ of $\mathcal{L}$ we understand a short exact sequence of Lie algebras

$$
0 \longrightarrow C \stackrel{i}{\longrightarrow} \tilde{\mathcal{L}} \stackrel{\pi}{\longrightarrow} \mathcal{L} \longrightarrow 0
$$

such that $C$ is in the center of $\tilde{\mathcal{L}}$.

(b) To verify that a covering is universal, it suffices to show the existence of a homomorphism from the covering to any central extension of $\mathcal{L}$. It is obvious from the universal property that, any two universal coverings are isomorphic as central extension.

(c) Any perfect Lie algebra $\mathcal{L}$ has a unique (up to isomorphism) universal covering $(\tilde{\mathcal{L}}, \pi)$ which is also perfect (see [11]). The kernel of $\pi$ is its second homology $\mathrm{H}_{2}(\mathcal{L}, \mathbb{F})$ with trivial coefficients (see [24, Theorem 7.9.2]).

Lemma 1.7. Let $(\tilde{\mathcal{L}}, \pi)$ be a central extension of perfect Lie algebra $\mathcal{L}$. Then

(i) $\tilde{\mathcal{L}}=[\tilde{\mathcal{L}}, \tilde{\mathcal{L}}]+\operatorname{ker} \pi$, and $\left([\tilde{\mathcal{L}}, \tilde{\mathcal{L}}],\left.\pi\right|_{[\tilde{\mathcal{L}}, \tilde{\mathcal{L}}]}\right)$ is a covering of $\mathcal{L}$,

(ii) $Z(\tilde{\mathcal{L}})=\pi^{-1}(Z(\mathcal{L}))$ and $\pi(Z(\tilde{\mathcal{L}}))=Z(\mathcal{L})$.

Proof. See [21, Lemma 1.5]. 


\section{Constructions for universal coverings of Lie tori}

In this section, we use Van der Kallen's method to construct universal coverings of Lie tori. These Lie algebras arise naturally in the construction of Extended affine Lie algebras.

Let $\mathcal{L}$ be a Lie torus. We consider the $\mathbb{F}$-submodule $J$ of $\mathbb{F}$-module $\mathcal{L} \otimes \mathcal{L}$ spanned by all elements of the form

$$
x \otimes x \quad \text { and } \quad x \otimes[y, z]+y \otimes[z, x]+z \otimes[x, y]
$$

for all $x, y, z \in \mathcal{L}$. Then $J$ can be thought of as a linear span of all homogeneous elements $\mathcal{L} \otimes \mathcal{L}$ of degree 2. Define $\tilde{\mathcal{L}}=(\mathcal{L} \otimes \mathcal{L}) / J$. For all $x, y$ in $\mathcal{L}, x \wedge y$ denotes the image of $x \otimes y$ under the canonical map, i.e., $x \wedge y=x \otimes y+J \in \tilde{\mathcal{L}}$. Note that we have the following identities in $\tilde{\mathcal{L}}$,

$$
x \wedge x=0, \quad x \wedge y=-y \wedge x \text { and } x \wedge[y, z]+y \wedge[z, x]+z \wedge[x, y]
$$

for all $x, y$ and $z$ in $\mathcal{L}$. By a straightforward computation, the $\mathbb{F}$-linear map $\mathcal{L} \otimes \mathcal{L} \longrightarrow[\mathcal{L}, \mathcal{L}]$ by $x \otimes y \longmapsto[x, y]$ vanishes on $J$ and hence descends to a $\mathbb{F}$-linear map

$$
\pi: \tilde{\mathcal{L}} \longrightarrow[\mathcal{L}, \mathcal{L}], \quad x \wedge y \longmapsto[x, y]
$$

for all $x, y \in \mathcal{L}$, with

$$
\operatorname{ker} \pi=\left\{\sum_{i} x_{i} \wedge y_{i} \mid \sum_{i}\left[x_{i}, y_{i}\right]=0\right\} .
$$

Now, we define a composition law $[\cdot, \cdot] \sim$ on $\tilde{\mathcal{L}}$ by

$$
\left[l_{1}, l_{2}\right]^{\sim}=\pi\left(l_{1}\right) \wedge \pi\left(l_{2}\right)
$$

for all $l_{1}, l_{2}$ in $\tilde{\mathcal{L}}$. The module $\tilde{\mathcal{L}}$ becomes an $\mathbb{F}$-algebra with respect to the product (2.3). Also by definition, $\pi$ is a homomorphism. In particular, we have

$$
\left[x \wedge y, x^{\prime} \wedge y^{\prime}\right]^{\sim}=[x, y] \wedge\left[x^{\prime}, y^{\prime}\right], \quad x, y, x^{\prime}, y^{\prime} \in \mathcal{L} .
$$

Lemma 2.5. Let $\mathcal{L}$ be a Lie torus. Then $\tilde{\mathcal{L}}$ is a Lie algebra with respect to product $[\cdot, \cdot] \sim$ in $(2.3)$.

Proof. It is clear that the product in $(2.3)$ on $\tilde{\mathcal{L}}$ is linear in each component. The anti-commutativity follows from (2.1). For the Jacobi identity, it is sufficient to verify it for elements of the spanning set $\{x \wedge y: x, y \in \mathcal{L}\}$. Consider three elements $l_{i}=x_{i} \wedge y_{i}$, where $i=1,2,3$ and $x_{i}, y_{i} \in \mathcal{L}$. By (2.3) and (2.4) we have

$$
\begin{aligned}
{\left[l_{1},\left[l_{2}, l_{3}\right]^{\sim}\right]^{\sim} } & =\pi\left(l_{1}\right) \wedge\left(\pi\left(l_{2}\right) \wedge \pi\left(l_{3}\right)\right) \\
& =\left[x_{1}, y_{1}\right] \wedge\left(\left[x_{2}, y_{2}\right] \wedge\left[x_{3}, y_{3}\right]\right) \\
& =\left[x_{1}, y_{1}\right] \wedge\left[\left[x_{2}, y_{2}\right],\left[x_{3}, y_{3}\right]\right] .
\end{aligned}
$$

By a similar computation and using $(2.1)$, we obtain $J a c\left(l_{1}, l_{2}, l_{3}\right)=0$. 
Proposition 2.6. Let $\mathcal{L}$ be a Lie torus. Then the pair $(\tilde{\mathcal{L}}, \pi)$ is a central extension of $\mathcal{L}$.

Proof. The $\mathbb{F}$-linear map $\pi$ is a homomorphism of Lie algebras. Indeed, by (2.2), (2.3) and (2.4) we have

$$
\begin{aligned}
\pi\left(\left[x \wedge y, x^{\prime} \wedge y^{\prime}\right]^{\sim}\right) & =\pi\left([x, y] \wedge\left[x^{\prime}, y^{\prime}\right]\right) \\
& =\left[[x, y],\left[x^{\prime}, y^{\prime}\right]\right] \\
& =\left[\pi(x \wedge y), \pi\left(x^{\prime} \wedge y^{\prime}\right] .\right.
\end{aligned}
$$

Now, since $\mathcal{L}$ is perfect $([\mathcal{L}, \mathcal{L}]=\mathcal{L}), \pi: \tilde{\mathcal{L}} \longrightarrow \mathcal{L}$ is a surjective Lie algebra homomorphism and also ker $\pi$ lies in the center of $\mathcal{L}$.

In this situation, we show that the central extension $(\tilde{\mathcal{L}}, \pi)$ of Lie torus $\mathcal{L}$ in Proposition 2.6, is a universal covering:

Theorem 2.7. Let $\mathcal{L}$ be a Lie torus. Then the pair $(\tilde{\mathcal{L}}, \pi)$ is a universal covering of $\mathcal{L}$.

Proof. Since $\mathcal{L}$ is a perfect Lie algebra, $\tilde{\mathcal{L}}$ is perfect. Then the central extension $(\tilde{\mathcal{L}}, \pi)$ is a covering of $\mathcal{L}$. To show that $(\tilde{\mathcal{L}}, \pi)$ is universal, it suffices to prove that there exists a homomorphism from $(\tilde{\mathcal{L}}, \pi)$ to any central extension of $\mathcal{L}$ (see Remark 1.6(b)). Suppose that $(\hat{\mathcal{L}}, \hat{\pi})$ is any central extension of $\mathcal{L}$. Then $\hat{\pi}$ is a surjective Lie algebra homomorphism, and since ker $\hat{\pi}$ lies in $Z(\hat{\mathcal{L}})$, we obtain the short exact sequence

$$
0 \longrightarrow \operatorname{ker} \hat{\pi} \stackrel{i}{\longrightarrow} \hat{\mathcal{L}} \stackrel{\hat{\pi}}{\longrightarrow} \mathcal{L} \longrightarrow 0 .
$$

Now, we choose a subspace $\mathcal{L}^{\prime}$ of $\hat{\mathcal{L}}$ such that $\hat{\pi}: \mathcal{L}^{\prime} \longrightarrow \mathcal{L}$ be an isomorphism of $\mathbb{F}$-vector space. Let $\alpha: \mathcal{L} \longrightarrow \mathcal{L}^{\prime}$ be the inverse map of $\hat{\pi}$. We will denote by $[\cdot, \cdot]$, the Lie product on $\hat{\mathcal{L}}$. Then for all $x, y \in \mathcal{L}$ we have

$$
\alpha([x, y])-[\alpha(x), \alpha(y) \hat{]} \in Z(\hat{\mathcal{L}}) .
$$

Our choice of $\mathcal{L}^{\prime}$ has led us to an $\mathbb{F}$-linear map

$$
\mathcal{L} \times \mathcal{L} \longrightarrow \hat{\mathcal{L}}, \quad(x, y) \longmapsto[\alpha(x), \alpha(y) \hat{]} .
$$

Hence by the universal property of the tensor product, we obtain an $\mathbb{F}$-linear map

$$
f: \mathcal{L} \otimes \mathcal{L} \longrightarrow \hat{\mathcal{L}}, \quad x \otimes y \longmapsto[\alpha(x), \alpha(y) \hat{]} .
$$

By (2.8) and the fact that $\hat{\mathcal{L}}$ is a Lie algebra over $\mathbb{F}$, this map vanishes on the $\mathbb{F}$-submodule $J$ of $\mathcal{L} \otimes_{\mathbb{F}} \mathcal{L}$. Thus we obtain an $\mathbb{F}$-linear map

$$
\psi: \tilde{\mathcal{L}} \longrightarrow \hat{\mathcal{L}}, \quad x \wedge y \longmapsto[\alpha(x), \alpha(y) \hat{]}
$$

for all $x, y \in \mathcal{L}$. We claim that $\psi$ is a Lie algebra homomorphism. For all $x \wedge y, x^{\prime} \wedge y^{\prime} \in \tilde{\mathcal{L}}$, by (2.8) and (2.9), we have

$$
\psi\left(\left[x \wedge y, x^{\prime} \wedge y^{\prime}\right]\right)=\psi\left([x, y] \wedge\left[x^{\prime}, y^{\prime}\right]\right)
$$




$$
\begin{aligned}
& =\left[\alpha([x, y]), \alpha\left(\left[x^{\prime}, y^{\prime}\right]\right) \hat{]}\right. \\
& =\left[[\alpha(x), \alpha(y)],\left[\alpha\left(x^{\prime}\right), \alpha\left(y^{\prime}\right)\right] \hat{]}\right. \\
& =\left[\psi(x \wedge y), \psi\left(x^{\prime} \wedge y^{\prime}\right)\right] .
\end{aligned}
$$

Next, we show that $\hat{\pi} \circ \psi=\pi$. Let $x, y \in \mathcal{L}$. Then

$$
\begin{aligned}
(\hat{\pi} \circ \psi)(x \wedge y) & =\hat{\pi}([\alpha(x), \alpha(y)]) \\
& =\hat{\pi}(\alpha([x, y])+z) \\
& =\hat{\pi}(\alpha([x, y])) \\
& =[x, y]+z^{\prime} \\
& =\pi(x \wedge y),
\end{aligned}
$$

where $z \in Z(\hat{\mathcal{L}})$ and $z^{\prime} \in Z(\mathcal{L})$. Thus $\psi$ is a Lie algebra homomorphism, which satisfies $\hat{\pi} \circ \psi=\pi$. By Definition 1.5, $\psi$ is a homomorphism from $(\tilde{\mathcal{L}}, \pi)$ to $(\hat{\mathcal{L}}, \hat{\pi})$. This completes the proof.

Corollary 2.10. Let $\mathcal{L}$ be a Lie torus. Then the $(\tilde{\mathcal{L}}, \pi)$ is a covering for all coverings of $\mathcal{L}$. In particular, $(\tilde{\mathcal{L}}, \pi)$ is a universal central extension of centerless Lie torus $\mathcal{L} / Z(\mathcal{L})$.

Proof. The first statement is obvious by the Remark 1.6(c) and Theorem 2.7. The last statement is now clear.

Let $\mathcal{L}$ be a Lie torus and $(\tilde{\mathcal{L}}, \pi)$ be its universal covering. We first recall the notion of lifting. An automorphism $\theta \in \operatorname{Aut}_{\mathbb{F}}(\mathcal{L})$ is said to lift to $\tilde{\mathcal{L}}$, if there exists an automorphism $\tilde{\theta} \in \operatorname{Aut}_{\mathbb{F}}(\tilde{\mathcal{L}})$ for which $\theta \circ \pi=\pi \circ \tilde{\theta}$.

Theorem 2.11 (lifting of automorphisms). Assume $\mathcal{L}$ is a centerless Lie torus and let $(\tilde{\mathcal{L}}, \pi)$ be its universal covering. Then

(i) The center $Z(\tilde{\mathcal{L}})$ of $\tilde{\mathcal{L}}$ is precisely the $\operatorname{ker}(\pi)$. Furthermore, the canonical map $\operatorname{Aut}_{\mathbb{F}}(\tilde{\mathcal{L}}) \longrightarrow \operatorname{Aut}_{\mathbb{F}}(\mathcal{L})$ is an isomorphism.

(ii) $\theta \in \operatorname{Aut}_{\mathbb{F}}(\mathcal{L})$ lifts to every central extension of $\mathcal{L}$ if and only if the lift $\tilde{\theta}$ of $\theta$ acts on $\operatorname{ker}(\pi)$ by scalar multiplication.

Proof. (i) See [23] for the proof. Another proof can be found in [21].

(ii) See [22, Lemma 2.7].

Proposition 2.12 (lifting of derivations). Let $(\tilde{\mathcal{L}}, \pi)$ be the universal covering of Lie torus $\mathcal{L}$. Then

$$
\operatorname{Der}_{\mathbb{F}}(\mathcal{L})=\left\{\delta \in \operatorname{Der}_{\mathbb{F}}(\tilde{\mathcal{L}}): \delta(\operatorname{ker}(\pi))=\operatorname{ker}(\pi)\right\} .
$$

In particular, $I \operatorname{Der}_{\mathbb{F}}(\tilde{\mathcal{L}}) \cong I \widetilde{\operatorname{Der}_{\mathbb{F}}}(\mathcal{L})$, i.e., $a d_{\tilde{\mathcal{L}}}(x \wedge y)=a \widetilde{d_{\mathcal{L}}[x, y]}$. Moreover, if $\mathcal{L}$ is a centerless Lie torus, then $\operatorname{Der}_{\mathbb{F}}(\mathcal{L}) \cong \operatorname{Der}_{\mathbb{F}}(\tilde{\mathcal{L}})$. 
Proof. Let $\delta \in \operatorname{Der}_{\mathbb{F}}(\mathcal{L})$. Consider the $\mathbb{F}$-linear map

$$
\delta \otimes \delta: \mathcal{L} \otimes \mathcal{L} \longrightarrow \mathcal{L} \otimes \mathcal{L}, \quad x \otimes y \longmapsto \delta(x) \otimes y+x \otimes \delta(y)
$$

for all $x, y \in \mathcal{L}$. This map leaves the submodule $J \subseteq \mathcal{L} \otimes \mathcal{L}$ invariant. Indeed, for all $x \in \mathcal{L}$ we have

$$
\begin{aligned}
(\delta \otimes \delta)(x \otimes x) & =\delta(x) \otimes x+x \otimes \delta(x) \\
& =(x+\delta(x)) \otimes(x+\delta(x))-(x \otimes x)-(\delta(x) \otimes \delta(x)) \in J .
\end{aligned}
$$

Also, for all $x, y \in \mathcal{L}$ a straightforward computation shows that

$$
\begin{aligned}
& (\delta \otimes \delta)(x \otimes[y, z]+y \otimes[z, x])+x \otimes[y, z]+z \otimes[x, y]) \\
= & (\delta(x) \otimes[y, z]+y \otimes[z, \delta(x)]+z \otimes[\delta(x), y]) \\
& +(x \otimes[\delta(y), z]+\delta(y) \otimes[z, x]+z \otimes[x, \delta(y)]) \\
& +(x \otimes[y, \delta(z)]+y \otimes[\delta(z), x]+\delta(z) \otimes[x, y]) \in J .
\end{aligned}
$$

Hence, $\delta \otimes \delta$ induces an $\mathbb{F}$-linear map

$$
\tilde{\delta}: \tilde{\mathcal{L}} \longrightarrow \tilde{\mathcal{L}}, \quad x \wedge y \longmapsto \delta(x) \wedge y+x \wedge \delta(y),
$$

which is a derivation on $\tilde{\mathcal{L}}$. Indeed, for all $l_{i}=x_{i} \wedge y_{i}$, where $i=1,2$ and $x_{i}, y_{i} \in \mathcal{L}$ we have

$$
\begin{aligned}
\tilde{\delta}\left(\left[l_{1}, l_{2}\right]\right)= & \tilde{\delta}\left(\pi\left(l_{1}\right) \wedge \pi\left(l_{2}\right)\right) \\
= & \delta\left(\pi\left(l_{1}\right)\right) \wedge \pi\left(l_{2}\right)+\pi\left(l_{1}\right) \wedge y \delta\left(l_{2}\right) \\
= & \delta\left[x_{1}, y_{1}\right] \wedge\left[x_{2}, y_{2}\right]+\left[x_{1}, y_{2}\right] \wedge \delta\left[x_{2}, y_{2}\right] \\
= & {\left[\delta\left(x_{1}\right), y_{1}\right] \wedge\left[x_{2}, y_{2}\right]+\left[x_{1}, \delta\left(y_{1}\right)\right] \wedge\left[x_{2}, y_{2}\right] } \\
& +\left[x_{1}, y_{2}\right] \wedge\left[\delta\left(x_{2}\right), y_{2}\right]+\left[x_{1}, y_{1}\right] \wedge\left[x_{2}, \delta\left(y_{2}\right)\right] \\
= & {\left[\delta\left(x_{1}\right) \wedge y_{1}+x_{1} \wedge \delta\left(y_{1}\right), x_{2} \wedge y_{2}\right]+\left[x_{1} \wedge y_{1}, \delta\left(x_{2}\right) \wedge y_{2}+x_{2} \wedge \delta\left(y_{2}\right)\right] } \\
= & {\left[\tilde{\delta}\left(x_{1} \wedge y_{1}\right), x_{2} \wedge y_{2}\right]+\left[x_{1} \wedge y_{1}, \tilde{\delta}\left(x_{2} \wedge y_{2}\right)\right] } \\
= & {\left[\tilde{\delta}\left(l_{1}\right), l_{2}\right]+\left[l_{1}, \tilde{\delta}\left(l_{2}\right)\right] . }
\end{aligned}
$$

It is clear that $\delta \circ \pi=\pi \circ \tilde{\delta}$. In particular, $\tilde{\delta}$ leaves $\operatorname{ker}(\pi)$ invariant, that is, $\tilde{\delta}$ acts on $\operatorname{ker}(\pi)$ by scalar multiplication. Then by Theorem 2.11 (ii), the map $\delta \longmapsto \tilde{\delta}$ is a Lie algebra homomorphism, which its kernel is zero on $[\mathcal{L}, \mathcal{L}]=\mathcal{L}$. This proves the first assertion. By the lifting Theorem of automorphisms, it is easily seen that for all $x, y \in \mathcal{L}$

$$
\widetilde{\operatorname{ad}_{\mathcal{L}}[x, y]}=\operatorname{ad}_{\tilde{\mathcal{L}}}(x \wedge y)
$$

whence $\left.\widetilde{\operatorname{ad}_{\mathcal{L}}(\pi(l)}\right)=\operatorname{ad}_{\tilde{\mathcal{L}}}(l)$ for all $l \in \tilde{\mathcal{L}}$, and so $\widetilde{\operatorname{ad}_{\mathcal{L}}}=\operatorname{IDer}_{\mathbb{F}}(\tilde{\mathcal{L}})$. If $\mathcal{L}$ is a centerless Lie torus, by Theorem 2.11(i) and the first part of this proposition, we get the result. 


\section{The structure of universal coverings of Lie tori}

In this section we give a completely description for the universal covering of a Lie $\Lambda$-torus of type $\Delta$. We will show that if $(\tilde{\mathcal{L}}, \pi)$ is a universal cover of $\mathcal{L}$, as in Section 2, then $\tilde{\mathcal{L}}$ is again a Lie $\Lambda$-torus of type $\Delta$.

Definition 3.1. Let $\Lambda$ be an abelian group and let $\mathcal{L}=\bigoplus_{\lambda \in \Lambda} \mathcal{L}^{\lambda}$ be a $\Lambda$-graded Lie algebra. By definition, a $\Lambda$-graded central extension of $\mathcal{L}$ is a $\Lambda$-graded Lie algebra $\mathcal{K}$ together with a central extension $\pi: \mathcal{K} \longrightarrow \mathcal{L}$ which is at the same time a homomorphism of $\Lambda$-graded Lie algebras and denoted by $(\mathcal{K}, \pi)$. A $\Lambda$ graded central extension $(\mathcal{K}, \pi)$ of $\mathcal{L}$ is a $\Lambda$-cover of $\mathcal{L}$ if $\pi: \mathcal{K} \longrightarrow \mathcal{L}$ is a covering, i.e., if $\mathcal{K}$ is perfect.

Proposition 3.2. Let $\mathcal{L}=\bigoplus_{\alpha \in \Delta} \bigoplus_{\lambda \in \Lambda} \mathcal{L}_{\alpha}^{\lambda}$ be a Lie $\Lambda$-torus of type $\Delta$ and $(\tilde{\mathcal{L}}, \pi)$ be its universal covering. Then $\tilde{\mathcal{L}}$ is a $(Q(\Delta) \oplus \Lambda)$-graded Lie algebra.

Proof. Consider $\mathcal{L}=\bigoplus_{\lambda \in \Lambda} \mathcal{L}^{\lambda}$ as a $\Lambda$-graded Lie algebra (see (1.4)). For $\lambda \in \Lambda$, let

$$
(\mathcal{L} \otimes \mathcal{L})^{\lambda}:=\bigoplus_{\mu \in \Lambda} \mathcal{L}^{\mu} \wedge \mathcal{L}^{\lambda-\mu}
$$

Then $\mathcal{L} \otimes \mathcal{L}=\bigoplus_{\lambda \in \Lambda}(\mathcal{L} \otimes \mathcal{L})^{\lambda}$ is a $\Lambda$-graded Lie algebra. So, the submodule $J \subseteq \mathcal{L} \otimes \mathcal{L}$ is a graded submodule of the $\Lambda$-graded module $\mathcal{L} \otimes \mathcal{L}$. Hence $\tilde{\mathcal{L}}=(\mathcal{L} \otimes \mathcal{L}) / J$ is $\Lambda$-graded with respect to the quotient grading. Namely, for $\lambda \in \Lambda$ we let $\tilde{\mathcal{L}}^{\lambda}:=\sum_{\mu \in \Lambda} \mathcal{L}^{\mu} \wedge \mathcal{L}^{\lambda-\mu}$ and define $\tilde{\mathcal{L}}=\bigoplus_{\lambda \in \Lambda} \tilde{\mathcal{L}}^{\lambda}$. By Definition $3.1, \pi\left(\mathcal{L}^{\lambda}\right) \subseteq \mathcal{L}^{\lambda}(\lambda \in \Lambda)$, so by $(2.3)$, we get

$$
\left[\tilde{\mathcal{L}}^{\lambda}, \tilde{\mathcal{L}}^{\mu}\right]=\pi\left(\tilde{\mathcal{L}}^{\lambda}\right) \wedge \pi\left(\tilde{\mathcal{L}}^{\mu}\right) \subseteq \sum_{\lambda, \mu \in \Lambda} \pi\left(\tilde{\mathcal{L}}^{\lambda}\right) \wedge \pi\left(\tilde{\mathcal{L}}^{\mu}\right) \subseteq \sum_{\lambda, \mu \in \Lambda} \mathcal{L}^{\lambda} \wedge \mathcal{L}^{\mu}=\tilde{\mathcal{L}}^{\lambda+\mu}
$$

Then $\tilde{\mathcal{L}}$ is a $\Lambda$-graded Lie algebra and that $\pi: \tilde{\mathcal{L}} \longrightarrow \mathcal{L}$ is a homomorphism of $\Lambda$-graded Lie algebras. Also, $\operatorname{ker}(\pi) \subseteq Z(\tilde{\mathcal{L}})$ is a $\Lambda$-graded submodule. Since $\tilde{\mathcal{L}}$ is perfect, $\pi$ is a covering and that $(\tilde{\mathcal{L}}, \pi)$ is a $\Lambda$-cover of $\mathcal{L}$. Now, we show that $\pi: \tilde{\mathcal{L}} \longrightarrow \mathcal{L}$ is a $(Q(\Delta) \oplus \Lambda)$-covering. Consider $\mathcal{L}=\bigoplus_{Q(\Delta)} \mathcal{L}_{\alpha}$ as a root graded Lie algebra (see (1.3)). Then $\mathcal{L}$ has a weight space decomposition with respect to the toral subalgebra $\mathcal{H} \subseteq \mathcal{L}_{0}^{0}$. Since $\mathcal{L}$ is perfect, we obtain that $\tilde{\mathcal{H}}:=\pi^{-1}(\mathcal{H})$ is a toral subalgebra of $\tilde{\mathcal{L}}$. It is also $\Lambda$-graded, i.e., for $\lambda \in \Lambda$, we let $\tilde{\mathcal{H}}^{\lambda}=\tilde{\mathcal{H}} \cap \tilde{\mathcal{L}}^{\lambda}$ and define $\tilde{\mathcal{H}}=\bigoplus_{\lambda \in \Lambda} \tilde{\mathcal{H}}^{\lambda}$. Note that, for all $0 \neq \lambda \in \Lambda$ we have

$$
\tilde{\mathcal{H}}^{\lambda} \subseteq \operatorname{ker}(\pi) \subseteq Z(\tilde{\mathcal{L}})
$$

For $\alpha \in Q(\Delta)$, let $\tilde{\mathcal{L}}_{\alpha}:=\sum_{\beta \in Q(\Delta)} \mathcal{L}_{\beta} \wedge \mathcal{L}_{\alpha-\beta}$ and define $\tilde{\mathcal{L}}=\bigoplus_{\alpha \in Q(\Delta)} \tilde{\mathcal{L}}_{\alpha}$. By (3.4), for all $\lambda \in \Lambda$ we have

$$
\left[\tilde{\mathcal{H}}, \tilde{\mathcal{L}}^{\lambda}\right]=\left[\bigoplus_{\lambda \in \Lambda} \tilde{\mathcal{H}}^{\lambda}, \tilde{\mathcal{L}}^{\lambda}\right] \subseteq \tilde{\mathcal{L}}^{\lambda}
$$


Hence, $\tilde{\mathcal{L}}^{\lambda}$ is an $\tilde{\mathcal{H}}$-submodule of $\tilde{\mathcal{L}}$ and so

$$
\tilde{\mathcal{L}}^{\lambda}=\bigoplus_{\alpha \in Q(\Delta)} \tilde{\mathcal{L}}_{\alpha}^{\lambda}, \quad \text { where } \quad \tilde{\mathcal{L}}_{\alpha}^{\lambda}:=\tilde{\mathcal{L}}_{\alpha} \cap \tilde{\mathcal{L}}^{\lambda} .
$$

That is, the root space decomposition of $\tilde{\mathcal{L}}$ with respect to $\tilde{\mathcal{H}}$ and the $\Lambda$-grading of $\tilde{\mathcal{L}}$ are compatible. So $\tilde{\mathcal{L}}=\bigoplus_{\alpha \in Q(\Delta)} \bigoplus_{\lambda \in \Lambda} \tilde{\mathcal{L}}_{\alpha}^{\lambda}$ is a $(Q(\Delta) \oplus \Lambda)$-graded Lie algebra and that $\pi: \tilde{\mathcal{L}} \longrightarrow \mathcal{L}$ is a $(Q(\Delta) \oplus \Lambda)$-covering.

Lemma 3.6. Let $\mathcal{L}=\bigoplus_{\alpha \in \Delta} \bigoplus_{\lambda \in \Lambda} \mathcal{L}_{\alpha}^{\lambda}$ be a Lie $\Lambda$-torus of type $\Delta$ and $(\tilde{\mathcal{L}}, \pi)$ be its universal covering. Then for all $\lambda \in \Lambda$ we have

$$
\tilde{\mathcal{L}}_{0}^{\lambda}=\sum_{\mu \in \Lambda, \alpha \in \Delta^{\times}}\left[\tilde{\mathcal{L}}_{\alpha}^{\mu}, \tilde{\mathcal{L}}_{-\alpha}^{\lambda-\mu}\right] .
$$

Proof. Let $\Upsilon$ be the right hand side of $(3.7)$. We know that $\tilde{\mathcal{L}}$ is perfect, then we have

$$
\tilde{\mathcal{L}}_{0}^{\lambda}=\left[\tilde{\mathcal{L}}_{0}^{\lambda}, \tilde{\mathcal{L}}_{0}^{\lambda}\right]+\Upsilon .
$$

We also observe that $Z(\tilde{\mathcal{L}})=\bigoplus_{\lambda \in \Lambda}\left(Z(\tilde{\mathcal{L}}) \cap \mathcal{L}_{0}^{\lambda}\right)$, and this implies that $\left.\pi\right|_{\tilde{\mathcal{L}}_{\alpha}^{\lambda}}$ is bijective for $\alpha \in \Delta^{\times}, \lambda \in \Lambda$. By this fact and by surjectivity of $\pi$, for all $\lambda \in \Lambda$ we have

$$
\begin{aligned}
\pi(\Upsilon) & =\sum_{\alpha \in \Delta^{\times}, \mu \in \Lambda} \pi\left(\tilde{\mathcal{L}}_{\alpha}^{\mu}\right) \wedge \pi(\tilde{\mathcal{L}})_{-\alpha}^{\lambda-\mu} \\
& =\sum_{\alpha \in \Delta^{\times}, \mu \in \Lambda}\left[\mathcal{L}_{\alpha}^{\mu}, \mathcal{L}_{-\alpha}^{\lambda-\mu}\right] .
\end{aligned}
$$

By (LT2) for $\mathcal{L}$, we get $\tilde{\mathcal{L}}_{0}^{\lambda} \subseteq \Upsilon+\operatorname{ker}(\pi)$. Since $\operatorname{ker}(\pi) \subseteq Z(\tilde{\mathcal{L}})$, it follows that

$$
\left[\tilde{\mathcal{L}}_{\alpha}^{\mu}, \tilde{\mathcal{L}}_{-\alpha}^{\lambda-\mu}\right] \subseteq[\Upsilon, \Upsilon \tilde{]}
$$

Now, we must show that $\Upsilon$ is a subalgebra of $\tilde{\mathcal{L}}$. By the Jacobi identity, for all $\lambda, \mu \in \Lambda$ and $\alpha \in \Delta^{\times}$

$$
\begin{aligned}
{\left[\tilde{\mathcal{L}}_{0}^{\lambda},\left[\tilde{\mathcal{L}}_{\alpha}^{\mu}, \tilde{\mathcal{L}}_{-\alpha}^{-\mu}\right] \tilde{]}\right.} & =\left[\left[\tilde{\mathcal{L}}_{0}^{\lambda}, \tilde{\mathcal{L}}_{\alpha}^{\mu} \tilde{]}, \tilde{\mathcal{L}}_{-\alpha}^{-\mu} \tilde{]}+\left[\tilde{\mathcal{L}}_{\alpha}^{\mu},\left[\tilde{\mathcal{L}}_{0}^{\lambda}, \tilde{\mathcal{L}}_{-\alpha}^{-\mu} \tilde{]}\right]\right.\right.\right. \\
& \subseteq\left[\tilde{\mathcal{L}}_{\alpha}^{\mu}, \tilde{\mathcal{L}}_{-\alpha}^{-\mu}\right]
\end{aligned}
$$

Proposition 3.8. For $\alpha \in \Delta^{\times}$and $\lambda \in \Lambda$ we have

(i) $\operatorname{dim} \tilde{\mathcal{L}}_{\alpha}^{\lambda} \leq 1$, with $\operatorname{dim} \tilde{\mathcal{L}}_{\alpha}^{0}=1$ if $\alpha \in \Delta_{\text {ind }}$.

(ii) If $\operatorname{dim} \tilde{\mathcal{L}}_{\alpha}^{\lambda}=1$, then there exists $\left(\tilde{x}_{\alpha}^{\lambda}, \tilde{y}_{\alpha}^{\lambda}\right) \in \tilde{\mathcal{L}}_{\alpha}^{\lambda} \times \tilde{\mathcal{L}}_{-\alpha}^{-\lambda}$ such that $\tilde{h}_{\alpha}^{\lambda}:=$ $\left[\tilde{x}_{\alpha}^{\lambda}, \tilde{y}_{\alpha}^{\lambda}\left\lceil\in \tilde{\mathcal{L}}_{0}^{0}\right.\right.$ acts on $\tilde{z}_{\beta}^{\mu} \in \tilde{\mathcal{L}}_{\beta}^{\mu}(\mu \in \Lambda, \beta \in \Delta)$ by $\left[\tilde{h}_{\alpha}^{\lambda}, \tilde{z}_{\beta}^{\mu}\right]=\langle\beta, \check{\alpha}\rangle \tilde{z}_{\beta}^{\mu}$.

Proof. (i) Since $\left.\pi\right|_{\tilde{\mathcal{L}}_{\alpha}^{\lambda}}$ is one to one, for all $\alpha \in \Delta^{\times}$and all $\lambda \in \Lambda$ we get the results.

(ii) Let $\operatorname{dim} \tilde{\mathcal{L}}_{\alpha}^{\lambda}=1\left(\lambda \in \Lambda, \alpha \in \Delta^{\times}\right)$. By part (i) we have $\operatorname{dim} \mathcal{L}_{\alpha}^{\lambda}=1$, so by (LT3)(ii) for $\mathcal{L}$, there exists $\left(x_{\alpha}^{\lambda}, y_{\alpha}^{\lambda}\right) \in \mathcal{L}_{\alpha}^{\lambda} \times \mathcal{L}_{-\alpha}^{-\lambda}$ such that $h_{\alpha}^{\lambda}:=\left[x_{\alpha}^{\lambda}, y_{\alpha}^{\lambda}\right] \in \mathcal{L}_{0}^{0}$ 
acts on $\mathcal{L}_{\beta}^{\mu}(\mu \in \Lambda, \beta \in \Delta)$ diagonally. For $\alpha \in \Delta^{\times}$and $\lambda \in \Lambda$, the map $\left.\pi\right|_{\tilde{\mathcal{L}}_{\alpha}^{\lambda}}$ is one to one. It follows that there exists $\left(\tilde{x}_{\alpha}^{\lambda}, \tilde{y}_{\alpha}^{\lambda}\right) \in \tilde{\mathcal{L}}_{\alpha}^{\lambda} \times \tilde{\mathcal{L}}_{-\alpha}^{-\lambda}$ such that they are mapped onto $\left(x_{\alpha}^{\lambda}, y_{\alpha}^{\lambda}\right) \in \mathcal{L}_{\alpha}^{\lambda} \times \mathcal{L}_{-\alpha}^{-\lambda}$ by $\pi$. Let

$$
\tilde{h}_{\alpha}^{\lambda}:=x_{\alpha}^{\lambda} \wedge y_{\alpha}^{\lambda}=\left[\tilde{x}_{\alpha}^{\lambda}, \tilde{y}_{\alpha}^{\lambda}\right] \in \tilde{\mathcal{L}}_{0}^{0} .
$$

By Proposition 3.2, we know that

$$
\tilde{\mathcal{L}}=\bigoplus_{\alpha \in Q(\Delta)} \bigoplus_{\lambda \in \Lambda} \tilde{\mathcal{L}}_{\alpha}^{\lambda}, \quad \text { where } \quad \tilde{\mathcal{L}}_{\alpha}^{\lambda}=\sum_{\beta \in \Delta, \mu \in \Lambda} \mathcal{L}_{\beta}^{\mu} \wedge \mathcal{L}_{\alpha-\beta}^{\lambda-\mu},
$$

with $\tilde{\mathcal{L}}_{\alpha}^{\lambda}=0$ if $\alpha \notin \Delta$. Now, for $\beta \in Q(\Delta), \gamma \in \Delta, \mu, \nu \in \Lambda$ and $v_{\gamma}^{\nu} \in$ $\mathcal{L}_{\gamma}^{\nu}, w_{\beta-\gamma}^{\mu-\nu} \in \mathcal{L}_{\beta-\gamma}^{\mu-\nu}$ we have

$$
\begin{aligned}
{\left[\tilde{h}_{\alpha}^{\lambda}, v_{\gamma}^{\nu} \wedge w_{\beta-\gamma}^{\mu-\nu}\right] } & =\left[x_{\alpha}^{\lambda} \wedge y_{\alpha}^{\lambda}, v_{\gamma}^{\nu} \wedge w_{\beta-\gamma}^{\mu-\nu}\right] \\
& =\left[x_{\alpha}^{\lambda}, y_{\alpha}^{\lambda}\right] \wedge\left[v_{\gamma}^{\nu}, w_{\beta-\gamma}^{\mu-\nu}\right] \\
& =h_{\alpha}^{\lambda} \wedge\left[v_{\gamma}^{\nu}, w_{\beta-\gamma}^{\mu-\nu}\right] \\
& =-v_{\gamma}^{\nu} \wedge\left[w_{\beta-\gamma}^{\mu-\nu}, h_{\alpha}^{\lambda}\right]-w_{\beta-\gamma}^{\mu-\nu} \wedge\left[h_{\alpha}^{\lambda} v_{\gamma}^{\nu}\right] \\
& =\langle\beta-\gamma, \check{\alpha}\rangle v_{\gamma}^{\nu} \wedge w_{\beta-\gamma}^{\mu-\nu}+\langle\gamma, \check{\alpha}\rangle v_{\gamma}^{\nu} \wedge w_{\beta-\gamma}^{\mu-\nu} \\
& =\langle\beta, \check{\alpha}\rangle v_{\gamma}^{\nu} \wedge w_{\beta-\gamma}^{\mu-\nu} .
\end{aligned}
$$

Remark 3.9. Let $\left(e_{\alpha}^{\lambda}, h_{\alpha}^{\lambda}, f_{\alpha}^{\lambda}\right)$ be an $\mathfrak{s l}_{2}^{(\alpha)}$-triple associated to $(\alpha, \lambda) \in \Delta^{\times} \times \Lambda$. By Proposition 3.8(ii), let

$$
\tilde{e}_{\alpha}^{\lambda}:=\frac{1}{2} h_{\alpha}^{\lambda} \wedge e_{\alpha}^{\lambda} \in \tilde{\mathcal{L}}_{\alpha}^{\lambda}, \quad \tilde{f}_{\alpha}^{\lambda}:=\frac{1}{2} f_{\alpha}^{\lambda} \wedge h_{\alpha}^{\lambda} \in \tilde{\mathcal{L}}_{-\alpha}^{-\lambda}, \quad \tilde{h}_{\alpha}^{\lambda}:=f_{\alpha}^{\lambda} \wedge e_{\alpha}^{\lambda} \in \tilde{\mathcal{L}}_{0}^{0} .
$$

They are mapped onto $e_{\alpha}^{\lambda}, h_{\alpha}^{\lambda}, f_{\alpha}^{\lambda}$ by $\pi$. Then the subalgebra $S_{\alpha}:=\mathbb{F} \tilde{e}_{\alpha}^{\lambda} \oplus$ $\mathbb{F} \tilde{h}_{\alpha}^{\lambda} \oplus \tilde{f}_{\alpha}^{\lambda}$ of $\tilde{\mathcal{L}}$ is isomorphic to the Lie algebra $\mathfrak{s l}_{2}(\mathbb{F})$.

Now, we summarize the above results in the following main theorem:

Theorem 3.10. Let $\mathcal{L}=\bigoplus_{\alpha \in \Delta} \bigoplus_{\lambda \in \Lambda} \mathcal{L}_{\alpha}^{\lambda}$ be a Lie $\Lambda$-torus of type $\Delta$ and $(\tilde{\mathcal{L}}, \pi)$ be its universal covering. Then $\tilde{\mathcal{L}}$ is a Lie $\Lambda$-torus of type $\Delta$.

Proof. By Propositions 3.2, 3.8 and Lemma 3.6, $\tilde{\mathcal{L}}$ satisfies in axioms (LT1)(LT3). To prove that $\tilde{\mathcal{L}}$ is a Lie $\Lambda$-torus of type $\Delta$, it remains to show that $\Lambda=\langle\operatorname{Supp} \tilde{\mathcal{L}}\rangle$. Suppose to contrary that $\tilde{\mathcal{L}}^{\lambda}=0$ for some $\lambda \in \Lambda$. Then $\mathcal{L}_{\alpha}^{\lambda}=0$ for any $\alpha \in Q(\Delta)$. Because of $\pi\left(\tilde{\mathcal{L}}_{\alpha}^{\lambda}\right)=0$, we get $\tilde{\mathcal{L}}_{\alpha}^{\lambda} \subseteq Z(\tilde{\mathcal{L}})$. Choose $\beta \in \Delta_{\text {ind }}$ such that $\langle\alpha, \breve{\beta}\rangle \neq 0$. By Remark 3.9, let $\left(\tilde{e}_{\beta}^{0}, \tilde{h}_{\beta}^{0}, \tilde{f}_{\beta}^{0}\right)$ be an $\mathfrak{s l}_{2}^{\beta}$-triple associated to $(\beta, \lambda) \in \Delta_{\text {ind }} \times \Lambda$. Then $\tilde{h}_{\beta}^{0}$ acts diagonally $\tilde{\mathcal{L}}_{\alpha}^{\lambda}$. That is, by Proposition 3.8(ii), for some $\gamma \in \Delta, \mu \in \Lambda$ and $x_{\gamma}^{\mu} \wedge y_{\alpha-\gamma}^{\lambda-\mu} \in \mathcal{L}_{\gamma}^{\mu} \wedge y_{\alpha-\gamma}^{\lambda-\mu}$ we have

$$
\left[\tilde{h}_{\beta}^{0}, x_{\gamma}^{\mu} \wedge y_{\alpha-\gamma}^{\lambda-\mu}\right]^{2}=\langle\alpha, \check{\beta}\rangle x_{\gamma}^{\mu} \wedge y_{\alpha-\gamma}^{\lambda-\mu} \text {. }
$$


Because of $\pi\left(\tilde{\mathcal{L}}_{\beta}^{\mu}\right)=0$, we have $\tilde{\mathcal{L}}_{\beta}^{\mu} \subseteq Z(\tilde{\mathcal{L}})$. It follows from (3.11) that $x_{\gamma}^{\mu} \wedge$ $y_{\alpha-\gamma}^{\lambda-\mu}=0$, which is a contradiction.

\section{References}

[1] B. Allison, S. Azam, S. Berman, Y. Gao, and A. Pianzola, Extended affine Lie algebras and their root systems, Mem. Amer. Math. Soc. 126 (1997), no. 603, 1-122.

[2] B. Allison, G. Benkart, and Y. Gao, Central extensions of Lie algebras graded by finite root systems, Math. Ann. 316 (2000), no. 3, 499-527.

[3] - Lie algebras graded by the root systems $B C_{r}, r \geq 2$, Mem. Amer. Math. Soc. 158 (2002), no. $751, \mathrm{x}+158$

4] S. Azam, Generalized reductive Lie algebars: coonections with extended affine Lie algebars and Lie tori, Canad. J. Math. 58 (2006), no. 2, 225-248.

[5] S. Azam and V. Khalili, Lie tori and their fixed point subalgebra, Algebra Colloq. 16 (2009), no. 3, 381-396.

[6] S. Azam, V. Khalili, and M. Yousofzadeh, Extended affine root system of type BC, J. Lie Theory 15 (2005), no. 1, 145-181.

[7] S. Azam, H. Yamane, and M. Yousofzadeh, A finite presentation of universal coverings of Lie tori, Publ. RIMS Kyoto Univ. 46 (2010), 507-548.

[8] G. Benkart and O. Smirnov, Lie algebras graded by the root system $B C_{1}$, J. Lie Theory 13 (2003), no. 1, 91-132.

[9] S. Berman and R. Moody, Lie algebras graded by finite root systems and the intersection matrix algebras of Slodowy, Invent. Math. 108 (1992), no. 2, 323-347.

[10] N. Bourbaki, Groupes et algèbres de Lie, Chap. 4-6, Hermann, Paris 1968.

[11] Y. Gao, Steinberg unitary Lie algebras and skew-dihedral homology, J. Algebra. 176 (1996), no. 1, 261-304.

[12] H. Garland, The arithmetic theory of loop groups, Inst. Hautes Études Sci. Publ. Math. (1980), no. 52, 5-136.

[13] V. Khalili, Extension data and their Lie algebras, Algebra Colloq. 18 (2011), no. 3, $461-474$.

[14] The core of a locally extended affine Lie algebras, Comm. Algebra 39 (2011), no. 10, 3646-3661.

[15] R. V. Moody and A. Pianzola, Lie Algebras with Triangular Decomposition, John Wiley, New York, 1995.

[16] J. Morita and Y. Yoshii, Locally extended affine Lie algebras, J. Algebra 301, (2006), no. $1,59-81$.

[17] K. H. Neeb, Universal central extensions of Lie groups, Acta Appl. Math. 73 (2002). no. 1-2, 175-219.

[18] E. Neher, Lie algebras graded by 3-graded root systems and Jordan pairs covered by grids, Amer. J. Math. 118 (1996), no. 2, 439-491.

[19] Lie tori, C. R. Math. Acad. Sci. Soc. R. Can. 26 (2004), no. 3, 84-89.

[20] _ Extended affine Lie algebras, C. R. Math. Acad. Sci. Soc. R. Can. 26 (2004), no. $3,90-96$

[21] An introduction to universal central extensions of Lie superalgebras, Groups, rings, Lie and Hopf algebras (St. John's, NF, 2001), 141-166, Math. Appl., 555, Kluwer Acad. Publ., Dordrecht, 2003.

[22] A. Pianzola, D. Prelet, and J. Sun, Descent constructions for central extensions of infinite dimensional Lie algebras, Manuscripta Math. 122 (2007), no. 2, 137-148.

[23] W. L. J. van der Kallen, Infinitesimacally Centrals Extension of Chevally Groups, Springer-Verlag, Berlin, 1973, Lecture Notes in Mathematics, Vol. 356, 1973. 
[24] C. Weibel, An Introduction to Homological Algebra, Cambridge Studies in Advanced Mathematics, 38. Cambridge University Press, Cambridge, 1994.

[25] Y. Yoshii, Root-graded Lie algebras with compatible grading, Comm. Algebra 20 (2001), no. 8, 3365-3391.

[26] - Root systems extended by an abelian group, and their Lie algebras, J. Lie theory, 14 (2004), no. 2, 371-394.

[27] L Lie tori-a simple characterization of extended affine Lie algebras, Publ. Res. Inst. Math. Sci. 42 (2006), no. 3, 739-762.

[28] M. Yousofzadeh, A presentation of Lie tori of type $B_{l}$, Publ. Res. Inst. Math. Sci. 44 (2008), no. 1, 1-44.

Department of Mathematics

FACUlTy OF SCIENCE

ARAK UNIVERSITY

ARAK 386156-8-8349, IRAN

E-mail address: V-Khalili@araku.ac.ir 